\title{
Identification of bovine-associated coagulase-negative staphylococci by matrix-assisted laser desorption/ionization time-of-flight mass spectrometry using a direct transfer protocol
}

\author{
M. Cameron, ${ }^{* 1}$ H. W. Barkema,† J. De Buck,† S. De Vliegher, $\ddagger$ M. Chaffer, ${ }^{*}$ J. Lewis,§ and G. P. Keefe* \\ *Department of Health Management, Atlantic Veterinary College, University of Prince Edward Island, Charlottetown, Prince Edward Island, \\ Canada C1A 4P3 \\ †Department of Production Animal Health, Faculty of Veterinary Medicine, University of Calgary, Calgary, Alberta, Canada T2N 1N4 \\ ¥M-team and Mastitis and Milk Quality Research Unit, Department of Reproduction, Obstetrics and Herd Health, Faculty of Veterinary Medicine, \\ Ghent University, Salisburylaan 133, 9820 Merelbeke, Belgium \\ §Department of Pathology and Microbiology, Atlantic Veterinary College, University of Prince Edward Island, Charlottetown, Prince Edward Island, \\ Canada C1A 4P3
}

\begin{abstract}
This study evaluated matrix-assisted laser desorption/ionization time-of-flight mass spectrometry (MALDI-ToF MS) for the identification of bovineassociated coagulase-negative staphylococci (CNS), a heterogeneous group of different species. Additionally, we aimed to expand the MALDI-ToF MS database with new reference spectra as required to fill the gaps within the existing commercial spectral library. A total of 258 isolates of CNS were used in the study, covering 16 different CNS species. The majority of the isolates were previously identified by $r p o B$ gene sequencing (n $=219$ ), and the remainder were identified by sequencing of $16 \mathrm{~S}$ rRNA, hsp60, or both rpoB and hsp60. The genotypic identification was considered the gold standard identification. All MALDI-ToF MS identifications were carried out using the direct transfer method. In a preliminary evaluation $(\mathrm{n}=32$ isolates; 2 of each species) with the existing commercial database, MALDIToF MS showed a typeability of $81 \%(26 / 32)$ and an accuracy of $96 \%(25 / 26)$. In the main evaluation $(\mathrm{n}=$ 226 isolates), MALDI-ToF MS with the existing commercial Biotyper (Bruker Daltonics Inc., Billerica, MA) database achieved a typeability of $92.0 \%(208 / 226)$ and an accuracy of $99.5 \%(207 / 208)$. Based on the assessment of the existing commercial database and prior knowledge of the species, a total of 13 custom reference spectra, covering 8 species, were created and added to the commercial database. Using the custom reference spectra expanded database, isolates were identified by MALDI-ToF MS with $100 \%$ typeability and $100 \%$ accuracy. Whereas the MALDI-ToF MS manufacturer's
\end{abstract}

Received September 20, 2016.

Accepted October 25, 2016.

${ }^{1}$ Corresponding author: mcameron@upei.ca cutoff for species-level identification is 2.000 , the reduction of the species level cutpoint to $\geq 1.700$ improved the species-level identification rates (from 64 to $92 \%$ for the existing commercial database) when classifying CNS isolates. Overall, MALDI-ToF MS using the direct transfer method was shown to be a highly reliable tool for the identification of bovine-associated CNS.

Key words: bovine coagulase-negative staphylococci, matrix-assisted laser desorption/ionization time-offlight mass spectrometry, species identification

\section{INTRODUCTION}

Mastitis remains an important disease of dairy cattle and causes substantial economic loss (Halasa et al., 2007; Ruegg, 2012). Pathogen-specific diagnosis is important for the management of bovine mastitis because modes of transmission and infection, as well as treatments, differ between different mastitis-causing organisms (Ruegg, 2012; Royster and Wagner, 2015). In many countries, as the prevalence of major mastitis pathogens such as Streptococcus agalactiae and Staphylococcus aureus has declined, a shift toward a greater detection of CNS has been observed (Makovec and Ruegg, 2003; Pitkälä et al., 2004; Piepers et al., 2007; Ruegg, 2012). According to a national-level study of the epidemiology of IMI in Canada conducted in 2007 and 2008, CNS was the most common bacterial group isolated among clinically normal quarters (recovered in $5.4 \%$ of quarters) and were approximately twice as common as the next most frequent organism (S. aureus, recovered in $2.4 \%$ of samples; Reyher et al., 2011).

The classification of CNS represents a group of species instead of a single pathogen species, as is common for most other mastitis etiologic diagnoses. Most diagnostic laboratories group these staphylococci together because of inaccuracy or cost of more explicit species 
determination (Zadoks and Watts, 2009; Ajitkumar et al., 2013). However, CNS species have vast differences in pathogenicity, ecology, and epidemiology, differences that arguably would rival those between other species of mastitis pathogens (Sampimon et al., 2011; Supré et al., 2011; Vanderhaeghen et al., 2014).

Matrix-assisted laser desorption/ionization timeof-flight mass spectrometry (MALDI-ToF MS) is a rapid, robust, and cost-effective technology that has revolutionized routine microbial diagnostics over the past half-decade (Kliem and Sauer, 2012). This technology relies on the generation of unique protein profiles (mass spectrum) captured from small amounts of bacterial colony material followed by software-assisted searching of a database containing known microbial spectra. The performance of MALDI-ToF MS systems is thus dependent upon the species coverage of the reference database. With regard to CNS, the commercially available Bruker MALDI Biotyper system employs a database that contains 32 different species of CNS (Biotyper reference database version 4.0.0.1 with 5,627 entries; Bruker Daltonics Inc., Billerica, MA). Included in this collection are Staphylococcus chromogenes, Staphylococcus epidermidis, Staphylococcus haemolyticus, Staphylococcus simulans, and Staphylococcus xylosus, which are reported to be the most prevalent species recovered in bovine milk in studies from around the world (Vanderhaeghen et al., 2015). In a small study of 108 bovine CNS isolates speciated by PCRRFLP, 95\% were correctly identified by MALDI-ToF MS (Tomazi et al., 2014). A limitation of that study was an uneven distribution of species such that of the 108 isolates of 11 species of CNS, $74.0 \%(\mathrm{n}=80)$ were S. chromogenes. Moreover, the reference methodology

Table 1. Collection of 16 species of CNS analyzed by matrix-assisted laser desorption/ionization time-of-flight mass spectrometry

\begin{tabular}{lrc}
\hline Species $^{1}$ & Number & Percentage of total \\
\hline Staphylococcus capitis & 3 & 1.2 \\
Staphylococcus chromogenes & 75 & 29.1 \\
Staphylococcus cohnii & 15 & 5.8 \\
Staphylococcus devriesei & 4 & 1.6 \\
Staphylococcus epidermidis & 34 & 13.2 \\
Staphylococcus equorum & 23 & 8.9 \\
Staphylococcus fleurettii & 5 & 1.9 \\
Staphylococcus gallinarum & 9 & 3.5 \\
Staphylococcus haemolyticus & 26 & 10.1 \\
Staphylococcus hyicus & 4 & 1.6 \\
Staphylococcus saprophyticus & 3 & 1.2 \\
Staphylococcus sciuri & 11 & 4.3 \\
Staphylococcus simulans & 11 & 4.3 \\
Staphylococcus succinus & 4 & 1.6 \\
Staphylococcus warneri & 7 & 2.7 \\
Staphylococcus xylosus & 24 & 9.3 \\
Total & 258 & 100 \\
\hline
\end{tabular}

${ }^{1}$ Determined by rpoB (219/258), rpoB and hsp60 (16/258), hsp60 $(12 / 258)$, or $16 S(11 / 258)$ gene sequencing.
(groEL PCR-RFLP) has only been validated against biochemical testing, which has been shown to suffer from a low accuracy (Vanderhaeghen et al., 2015). Whereas the results of this previous study show promise, further studies of the evaluation of MALDI-ToF MS for bovine-associated CNS using a greater number of isolates originating from bovine-associated habitats such as milk and better representation of species are required. The results of MALDI-ToF MS validation research will be valuable to the increasing number of diagnostic laboratories that are adopting MALDI-ToF MS for microbial identification. The main objectives of this study were therefore to evaluate MALDI-ToF MS for the identification of bovine-associated CNS and to expand the MALDI-ToF MS database with new reference spectra as required to fill the gaps within the existing commercially available spectral library.

\section{MATERIALS AND METHODS}

\section{Bacterial Isolates and Culture}

One hundred and one isolates belonging to the Mastitis Pathogen Culture Collection (MPCC) of the Canadian Bovine Mastitis and Milk Quality Research Network (Ajitkumar et al., 2013) and 157 isolates sourced from Ghent University, Belgium (Supré et al., 2009), were used in the study, covering 16 different CNS species (Table 1). Isolates were mainly recovered from milk samples $(\mathrm{n}=241 ; 93 \%)$, with the remainder isolated from teat apex swabs. The majority of the isolates were previously identified by $r p o B$ gene sequencing with $\geq 94.0 \%$ sequence homology to the GenBank database $(\mathrm{n}=238)$; the remainder were identified by sequencing of $16 \mathrm{~S}$ rRNA (with $\geq 98.7 \%$ homology; $\mathrm{n}=12$ ) or $h s p 60$ (with $\geq 97 \%$ homology; $n=8$; Mellmann et al., 2006; Supré et al., 2009; Ajitkumar et al., 2013).

Stock isolates, which were stored at $-80^{\circ} \mathrm{C}$, were thawed at room temperature for $15 \mathrm{~min}$. Columbia agar plates containing $5 \%$ sheep blood were streaked with a $1-\mu \mathrm{L}$ sterile disposable loop to obtain isolated colonies. Plates were incubated for 18 to $24 \mathrm{~h}$ at $35^{\circ} \mathrm{C}$. The following day, cultures were examined for growth and purity. Single colonies were sub-cultured onto blood agars and incubated for 18 to $24 \mathrm{~h}$ at $35^{\circ} \mathrm{C}$.

\section{MALDI-ToF Mass Spectrometry}

Sample Preparation. All isolate classifications were carried out using the direct transfer method (MALDI Biotyper 3.1 User Manual, Bruker Daltonics Inc.). Briefly, a single-use, 15 -cm sterile wooden applicator stick was used to lift material from a well-isolated bacterial colony followed by smearing a thin film of 
colony material onto a ground steel MSP 96-spot target (Bruker Daltonics Inc.). The spots were allowed to air dry at room temperature. Subsequently, the spots were overlaid with $1.0 \mu \mathrm{L}$ of a saturated solution of $\alpha$-cyano4-hydroxycinnamic acid matrix in 50\% acetonitrile, $47.5 \%$ water, and $2.5 \%$ trifluoroacetic acid (Sigma-Aldrich Canada Inc., Oakville, ON, Canada) using singleuse pipette tips and air-dried at room temperature.

MALDI-ToF MS Classification of CNS and Data Interpretation. All targets were calibrated using Bacterial Test Standard (Bruker Daltonics Inc.) and included S. aureus ATCC 29213 and Escherichia coli ATCC 25922 control classification samples in duplicate. To confirm that target cleaning was effective and no residual bacterial material from a previous run remained, one spot on each target contained only matrix with no bacterial sample. All bacterial spectral captures and classifications were carried out using a Bruker Daltonics Research Use Only microbial classification platform that included a Microflex LT mass spectrometer, flexControl software (version 3.4), MALDI Biotyper Real Time Classification (RTC), and Offline Classification (OC; version 3.1) with a 5,627 Main SPectrum (MSP) reference database library (MBT-BDAL-5627). All spectra reviews were carried out using flexAnalysis software (version 3.4, Bruker Daltonics Inc.). The MALDI Biotyper RTC was carried out according to MALDI Biotyper 3.1 User Manual. FlexControl settings were medium mass range $(1,960-21,200 \mathrm{~m} / \mathrm{z})$, detector gain set to $8.6 \times(3,227 \mathrm{~V})$, sample and digitizer settings at $0.50 \mathrm{GS} / \mathrm{s}$, and ion source 1 and 2 values were 20.12 and $18.25 \mathrm{kV}$, respectively. Each sample spectrum was summed from a maximum of 240 shots accumulated from a minimum of 6 raster points using a spiral-small laser movement pattern.

After acquisition of a sample's mass spectrum, the Biotyper software compares the spectrum to the reference spectra contained in the database. The software then displays matching identifications and computes a score ranging from 0 and 3 , indicating the degree of similarity between the sample's spectrum and the reference spectrum. Identification scores were interpreted as per manufacturer's recommendations as follows: a score of 2.000 to 3.000 was acceptable species-level identification, a score of 1.700 to 1.999 indicated identification to the genus level, and a score of $<1.700$ was considered no reliable identification.

Preliminary assessment of the Bruker database consisted of MALDI-ToF MS identification of 2 isolates of each species $(\mathrm{n}=32)$ run in 6 replicates, for a total of 12 repetitions per species, for a precision estimate as is routinely recommended for quality assurance of diagnostic laboratory equipment (Bellamy and Olexson, 2000). Subsequently, the remainder of the isolates $(\mathrm{n}=$
226; designated as the main classification) were run in duplicate (Figure 1). Isolates not used in the preliminary analysis and failing to achieve a score of $\geq 1.700$ (no reliable identification) on either of the 2 replicates were re-classified with a maximum of 4 replicates per isolate.

Custom Main Spectrum Creation. For 6 species of CNS (Staphylococcus cohnii, Staphylococcus devriesei, Staphylococcus fleurettii, Staphylococcus gallinarum, Staphylococcus warneri, and Staphylococcus xylosus) with isolates that failed to be identified by MALDI-ToF MS, in either the preliminary run or the main classification, custom MSP were created and added to the Bruker database. Additional MSP for $S$. chromogenes were generated because only one MSP for $S$. chromogenes was present in the existing commercial database. Previous genotypic examination of isolates of $S$. chromogenes contained in the MPCC identified 3 genotypes; therefore, a decision was made to capture each genotype with a unique MSP. The decision to add an MSP for $S$. haemolyticus was made after examination of the spectra acquired from the samples identified variations in the spectral profiles, suggesting that additional reference spectra would assist with the identification of isolates of these species.

A total of 13 custom MSP covering the 8 species listed above (see Supplemental Table S1; https://doi. org/10.3168/jds.2016-12020) were created as described below (MALDI Biotyper 3.1 User Manual; Bruker Daltonics Custom MSP and Library Creation, revision I, 2015). The isolates used to create the MSP belonged to the collection of 258 , with $6 / 13$ classified in the preliminary analysis and 7/13 classified against the existing commercial database in the main classification. Except for the isolates of $S$. devriesei and S. gallinarum, which had no entries in the existing commercial database, the isolates used for MSP creation were accurately identified by the MALDI-ToF MS. Briefly, single colonies from rроB sequence verified Staphylococcus spp. were sub-cultured to blood agar and incubated overnight at 35 to $37^{\circ} \mathrm{C}$. Well-isolated colonies $(\mathrm{n}=12-16)$ were transferred to individual 1.5-mL Eppendorf Safe-Lock Tubes (Sigma-Aldrich Canada Inc.) containing $300 \mu \mathrm{L}$ of Chromasolv LC-MS grade water (Sigma-Aldrich Canada Inc.) using sterile $1.0 \mu \mathrm{L}$ disposable plastic inoculating loops. Tubes were vortexed $2 \times 30$ s to create a homogeneous suspension followed by the addition of $900 \mu \mathrm{L}$ of $100 \%$ alcohol and an additional $60 \mathrm{~s}$ of vortex mixing. Tubes were centrifuged for $2 \mathrm{~min}$ at 17,000 $\times g$ at room temperature, and without disturbing the bacterial cell pellet, supernatants were aspirated and discarded. Tubes were centrifuged again for $1 \mathrm{~min}$ at $17,000 \times g$ at room temperature, and remaining etha$\mathrm{nol} /$ water was carefully aspirated using a micropipette. 


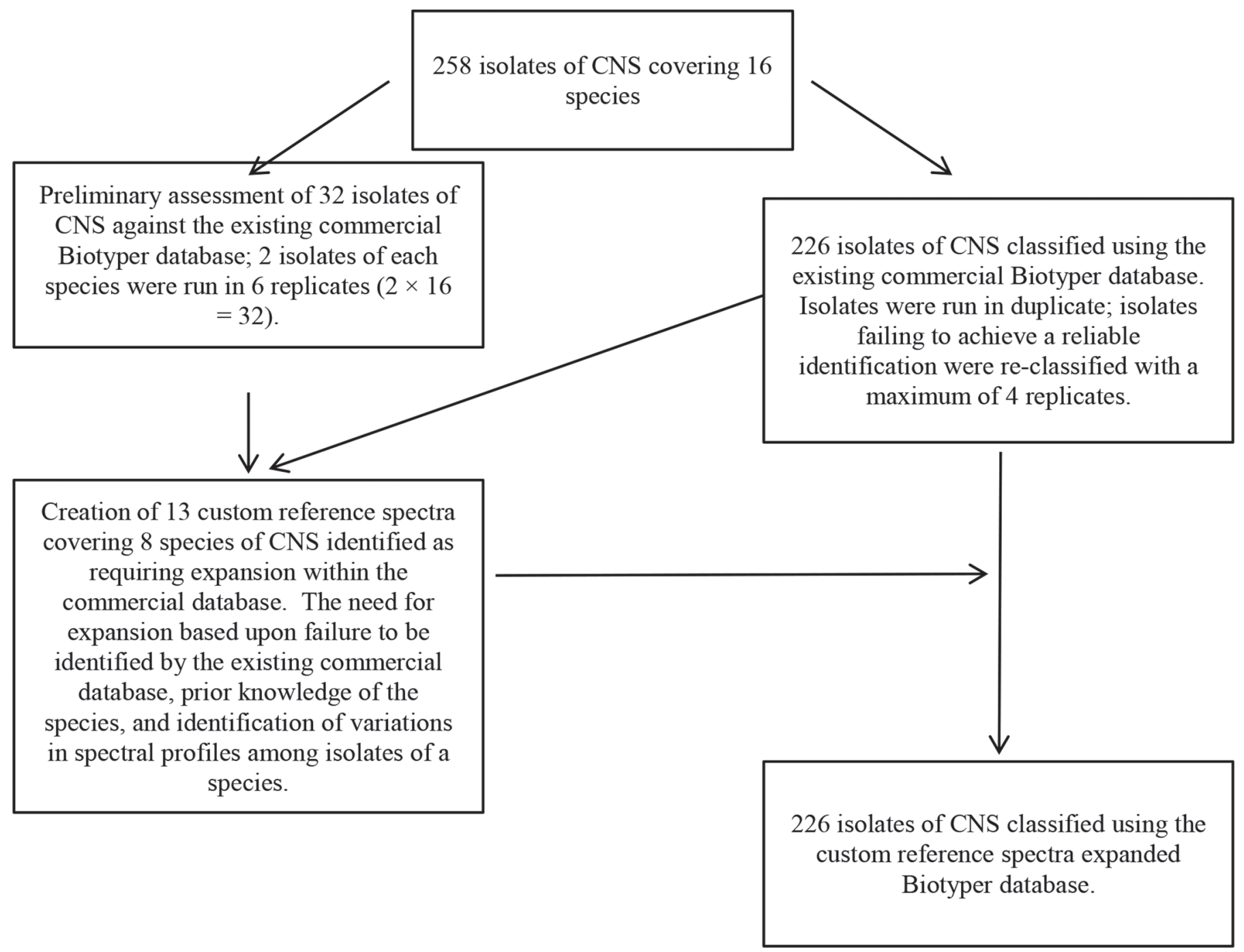

Figure 1. Flow of 258 bovine-associated isolates of 16 species of CNS through matrix-assisted laser desorption/ionization time-of-flight mass spectrometry classification against the existing commercial Biotyper (Bruker Daltonics Inc., Billerica, MA) database and a custom reference spectra expanded Biotyper database. Evaluation consisted of a preliminary assessment using 32 CNS isolates against only the existing commercial database, followed by a main assessment using 226 against both the existing commercial and expanded databases.

Cell pellets were dried in a biological safety cabinet for 2 to $3 \mathrm{~min}$ before the addition of an appropriate volume of fresh $70 \%$ formic acid. Optimal formic acid volumes were determined for some samples by carrying out a pre-extraction volume optimization step using a pooled colony sample followed by spectrum acquisition using Biotyper RTC and review of classification scores as well as spectra quality assessment using flexAnalysis. Samples were mixed by pipetting 10 to 12 times followed by brief mixing with a vortex mixer. After 6 to $7 \mathrm{~min}$, an equal volume of $100 \%$ acetonitrile was added to each sample and mixed carefully, with a micropipettor, 6 to 7 times followed by 2 to $4 \mathrm{~s}$ of vortex mixing. Samples were centrifuged for $2 \mathrm{~min}$ at $17,000 \times g$ at room temperature. Typically, $1.0-\mu \mathrm{L}$ volumes of supernatant were placed on a 96-spot ground steel target and allowed to dry at room temperature before being overlaid with $1.0 \mu \mathrm{L}$ of $\alpha$-cyano-4-hydroxycinnamic acid matrix.

Following calibration with Bacterial Test Standard, spectra from each sample were captured in triplicate using flexControl parameters described above. In some cases, duplicate and triplicate spectrum acquisitions were carried out using different laser movement patterns to minimize spot re-sampling and global peak intensity reduction. All spectra files were copied to spectra review folders and opened in flexAnalysis. Spectra were smoothed and baselines subtracted before assessment. 
Peak lists for representative spectra were generated using flexControl method processing parameters (see above). Low intensity and outlier spectra were identified until a minimum of 20 to 24 individual spectra were identified for custom MSP creation. Raw spectra from origin files were retrieved into Biotyper OC and custom MSP were created using the MSP Dendrogram Creation Standard Method (version 1.4) and default parameter settings. Quality checks for all custom MSP included classification of MSP creation spectra against the new MSP, new MSP classification against Bruker database entries as well as the new MSP, and review of new MSP peak lists to ensure that peak intensities and frequencies were satisfactory. Low intensity or low frequency peaks were reviewed using representative spectra in flexAnalysis. In cases where intensities or frequencies did not meet minimum standards, the formic acid extraction and spectrum acquisition was reviewed, re-optimized, and repeated.

Following the creation of custom MSP, archived spectra from the analysis of the 226 isolates (main classification) were re-classified using Biotyper OC identification standard method (version 1.1 with default parameter settings) and with the 13 CNS custom MSP in addition to the Bruker MBT-BDAL-5627 reference MSP database library (Figure 1).

\section{Data Analysis}

The highest MALDI-ToF Biotyper classification score among the replicates of an isolate was considered the final identification and score. The performance of MALDI-ToF MS was evaluated in terms of its typeability and accuracy. In this case, typeability refers to the ability of MALDI-ToF MS to assign a species name to a sample, and accuracy is the ability of MALDI-ToF MS to obtain the correct species name for a sample, considering the gene sequencing identification to be the gold standard identity of an isolate. Discordant identifications were defined as identifications by MALDI-ToF MS that did not match the gold standard species.

\section{Resolution of Discordant Identifications}

A total of 18 isolates with discordant identification as described above were re-submitted for $h s p 60$ and rpoB sequencing; an additional discordant isolate was submitted for $h s p 60$ sequencing only. To ensure that the sample analyzed by MALDI-ToF MS was the same as the sample submitted for sequencing, for each sample, a single isolated colony was spotted onto the target in duplicate and a portion of that same colony was streaked onto a new blood agar plate to create bacterial colonies for DNA extraction and sequencing.

\section{RESULTS}

\section{Preliminary Assessment}

According to preliminary assessment, MALDI-ToF MS equipped with the existing commercial database showed a typeability of $81 \%(26 / 32)$ and achieved an accuracy of $96 \%(25 / 26$; Table 2$)$. The 6 isolates that were not identified were $S$. cohnii, $S$. devriesei, and $S$. gallinarum. A single $S$. hyicus isolate was misidentified as $S$. chromogenes (identification score 2.025); however, the next closest match was $S$. hyicus (identification score 1.803). As per the manufacturer's score interpretation criteria, $59 \%(19 / 32)$ of the isolates achieved confident species level scores (i.e., $\geq 2.000$; Table 2 ).

\section{Main Classification with Existing Commercial Biotyper Database}

A breakdown of the MALDI-ToF MS classification results under the existing commercial database by species is contained in Table 3. Overall, using the existing commercial Biotyper database, isolates were identified by MALDI-ToF MS with a typeability of $92.0 \%(208 / 226)$ and an accuracy of $99.5 \%(207 / 208)$. In addition to the 3 species without identification detected in the preliminary assessment, one isolate of $S$. warneri and 2 isolates of $S$. xylosus were not typed by the existing commercial database. A single $S$. fleurettii isolate was misidentified as S. sciuri (identification score 1.907); however, the next closest match was $S$. fleurettii (identification score 1.892). An additional case of poor discrimination occurred with a second isolate of $S$. fleurettii, which was correctly identified with a score of 1.792 , but the next closest match was $S$. sciuri with a score of 1.755 . According to the manufacturer's score interpretation criteria, $63.7 \%(144 / 226)$ of the isolates achieved reliable species level scores (i.e., $\geq 2.000$; Table 3 ). The median absolute difference in identification score between replicate classifications of an isolate was 0.081 , with an interquartile range of $0.104 ; 10 \%(22 / 226)$ of samples required 2 spots to achieve an identification (i.e., one replicate was unidentified, but the second replicate achieved identification); $12 \%(26 / 226)$ isolates were reclassified with a maximum of 4 replicates per isolate because of failure to achieve an identification score $\geq 1.700$ on the first round of duplicate classifications.

\section{Main Classification with Custom MSP Expanded Biotyper Database}

A breakdown of the MALDI-ToF MS results using the custom MSP expanded database by species is presented in Table 4. Overall, MALDI-ToF MS using the 
Table 2. Identification of 32 CNS isolates by matrix-assisted laser desorption/ionization time-of-flight mass spectrometry (MALDI-ToF MS) compared with rpoB (29/32), $16 S(2 / 32)$, or hsp60 (1/32) gene sequencing as the reference method as a preliminary assessment of the Bruker Biotyper (Bruker Daltonics Inc., Billerica, MA) database

\begin{tabular}{|c|c|c|c|c|c|c|c|c|}
\hline \multirow[b]{2}{*}{ CNS species } & \multicolumn{2}{|c|}{ Misidentified $^{1}$} & \multicolumn{2}{|c|}{$\begin{array}{r}\text { Unidentified; } \\
\text { score }<1.700^{2}\end{array}$} & \multicolumn{2}{|c|}{$\begin{array}{l}\text { Correct species; } \\
\text { score } 1.700 \text { to } \\
\quad<2.000^{3}\end{array}$} & \multicolumn{2}{|c|}{$\begin{array}{l}\text { Correct species } \\
\text { score } \geq 2.000^{4}\end{array}$} \\
\hline & $\mathrm{n}$ & $\%$ & $\mathrm{n}$ & $\%$ & $\mathrm{n}$ & $\%$ & $\mathrm{n}$ & $\%$ \\
\hline Staphylococcus capitis & 0 & 0 & 0 & 0 & 0 & 0 & 2 & 100 \\
\hline Staphylococcus chromogenes & 0 & 0 & 0 & 0 & 0 & 0 & 2 & 100 \\
\hline Staphylococcus cohnii & 0 & 0 & 2 & 100 & 0 & 0 & 0 & 0 \\
\hline Staphylococcus devriesei & 0 & 0 & 2 & 100 & 0 & 0 & 0 & 0 \\
\hline Staphylococcus epidermidis & 0 & 0 & 0 & 0 & 0 & 0 & 2 & 100 \\
\hline Staphylococcus equorum & 0 & 0 & 0 & 0 & 0 & 0 & 2 & 100 \\
\hline Staphylococcus fleurettii & 0 & 0 & 0 & 0 & 2 & 100 & 0 & 0 \\
\hline Staphylococcus gallinarum & 0 & 0 & 2 & 100 & 0 & 0 & 0 & 0 \\
\hline Staphylococcus haemolyticus & 0 & 0 & 0 & 0 & 1 & 50 & 1 & 50 \\
\hline Staphylococcus hyicus & 1 & 50 & 0 & 0 & 0 & 0 & 1 & 50 \\
\hline Staphylococcus saprophyticus & 0 & 0 & 0 & 0 & 1 & 50 & 1 & 50 \\
\hline Staphylococcus sciuri & 0 & 0 & 0 & 0 & 0 & 0 & 2 & 100 \\
\hline Staphylococcus simulans & 0 & 0 & 0 & 0 & 0 & 0 & 2 & 100 \\
\hline Staphylococcus succinus & 0 & 0 & 0 & 0 & 2 & 100 & 0 & 0 \\
\hline Staphylococcus warneri & 0 & 0 & 0 & 0 & 2 & 100 & 0 & 0 \\
\hline Staphylococcus xylosus & 0 & 0 & 0 & 0 & 0 & 0 & 2 & 100 \\
\hline Total & 1 & 3 & 6 & 19 & 8 & 25 & 17 & 53 \\
\hline
\end{tabular}

${ }^{1}$ MALDI-ToF MS species is not the same as the reference.

${ }^{2}$ Manufacturer's score interpretation: no reliable identification.

${ }^{3}$ Manufacturer's score interpretation: reliable identification to the genus level.

${ }^{4}$ Manufacturer's score interpretation: reliable identification to the species level.

expanded database achieved a typeability of $100 \%$ and guish isolates of this species from isolates of S. sciuri. an accuracy of $100 \%$. With addition of an MSP for $S$. The 2 isolates of $S$. fleurettii poorly discriminated from fleurettii, MALDI-ToF MS was able to better distin- S. sciuri by the existing commercial database were cor-

Table 3. Identification of $226 \mathrm{CNS}$ isolates by matrix-assisted laser desorption/ionization time-of-flight mass spectrometry (MALDI-ToF MS) and the commercial Biotyper (Bruker Daltonics Inc., Billerica, MA) database compared with rpoB (190/226), rpoB and hsp60 (16/226), hsp60 $(11 / 226)$, or $16 S(9 / 226)$ gene sequencing

\begin{tabular}{|c|c|c|c|c|c|c|c|c|}
\hline \multirow[b]{2}{*}{ CNS species } & \multicolumn{2}{|c|}{ Misidentified $^{1}$} & \multicolumn{2}{|c|}{$\begin{array}{l}\text { Unidentified; score } \\
<1.700^{2}\end{array}$} & \multicolumn{2}{|c|}{$\begin{array}{c}\text { Correct species; score } \\
1.700 \text { to }<2.000^{3}\end{array}$} & \multicolumn{2}{|c|}{$\begin{array}{c}\text { Correct species; score } \geq \\
2.000^{4}\end{array}$} \\
\hline & $\mathrm{n}$ & $\%$ & $\mathrm{n}$ & $\%$ & $\mathrm{n}$ & $\%$ & $\mathrm{n}$ & $\%$ \\
\hline Staphylococcus capitis & 0 & 0 & 0 & 0 & 0 & 0 & 1 & 100 \\
\hline Staphylococcus chromogenes & 0 & 0 & 0 & 0 & 16 & 22 & 57 & 78 \\
\hline Staphylococcus cohnii & 0 & 0 & 6 & 46 & 6 & 46 & 1 & 8 \\
\hline Staphylococcus devriesei & 0 & 0 & 2 & 100 & 0 & 0 & 0 & 0 \\
\hline Staphylococcus epidermidis & 0 & 0 & 0 & 0 & 0 & 0 & 32 & 100 \\
\hline Staphylococcus equorum & 0 & 0 & 0 & 0 & 4 & 19 & 17 & 81 \\
\hline Staphylococcus fleurettii & 1 & 33 & 0 & 0 & 2 & 67 & 0 & 0 \\
\hline Staphylococcus gallinarum & 0 & 0 & 7 & 100 & 0 & 0 & 0 & 0 \\
\hline Staphylococcus haemolyticus & 0 & 0 & 0 & 0 & 20 & 83 & 4 & 17 \\
\hline Staphylococcus hyicus & 0 & 0 & 0 & 0 & 2 & 100 & 0 & 0 \\
\hline Staphylococcus saprophyticus & 0 & 0 & 0 & 0 & 1 & 100 & 0 & 0 \\
\hline Staphylococcus sciuri & 0 & 0 & 0 & 0 & 0 & 0 & 9 & 100 \\
\hline Staphylococcus simulans & 0 & 0 & 0 & 0 & 2 & 22 & 7 & 78 \\
\hline Staphylococcus succinus & 0 & 0 & 0 & 0 & 2 & 100 & 0 & 0 \\
\hline Staphylococcus warneri & 0 & 0 & 1 & 20 & 1 & 20 & 3 & 60 \\
\hline Staphylococcus xylosus & 0 & 0 & 2 & 9 & 7 & 32 & 13 & 59 \\
\hline Total & 1 & 0 & 18 & 8 & 63 & 28 & 144 & 64 \\
\hline
\end{tabular}

${ }^{1}$ MALDI-ToF MS species is not the same as the reference.

${ }^{2}$ Manufacturer's score interpretation: no reliable identification.

${ }^{3}$ Manufacturer's score interpretation: reliable identification to the genus level.

${ }^{4}$ Manufacturer's score interpretation: reliable identification to the species level. 
Table 4. Identification of 226 CNS isolates by matrix-assisted laser desorption/ionization time-of-flight mass spectrometry (MALDI-ToF MS) and the in-house expanded Biotyper database compared with rpoB (190/226), rpoB and hsp60 (16/226), hsp60 (11/226), or 16S (9/226) gene sequencing

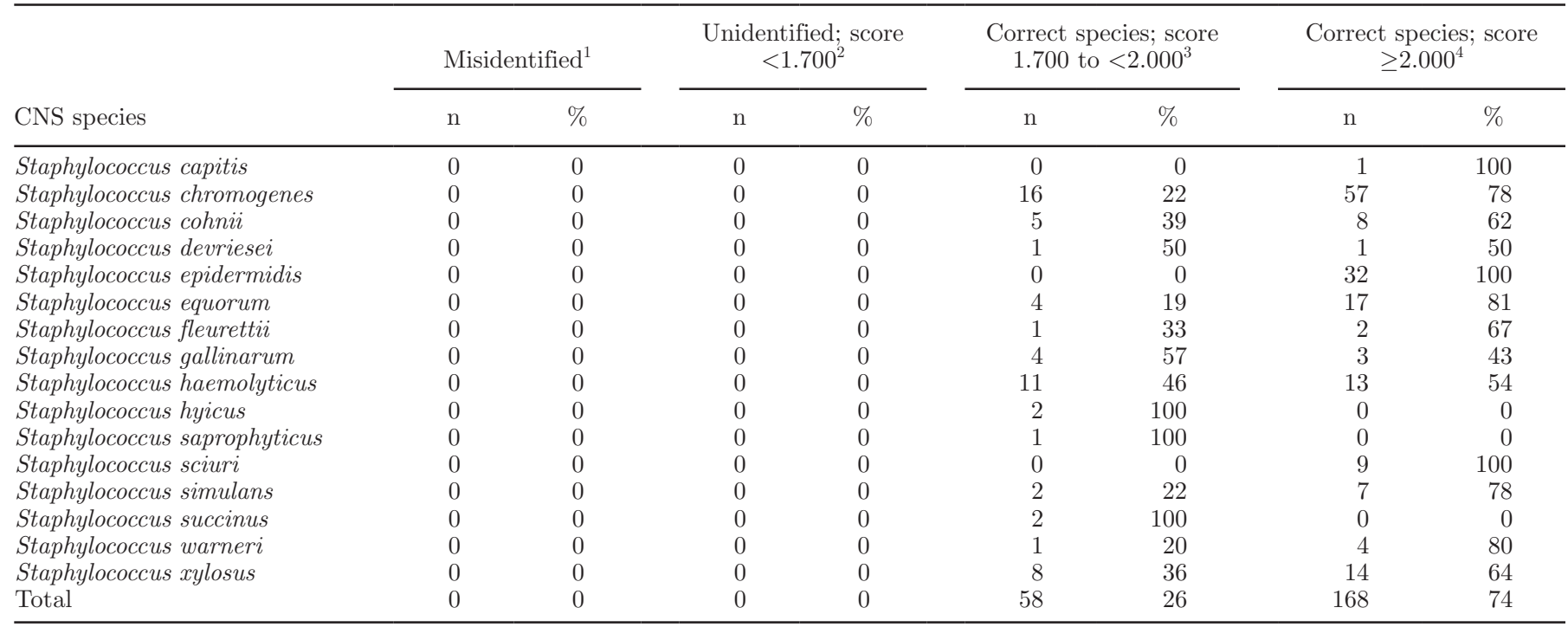

${ }^{1}$ MALDI-ToF MS species is not the same as the reference.

${ }^{2}$ Manufacturer's score interpretation: no reliable identification.

${ }^{3}$ Manufacturer's score interpretation: reliable identification to the genus level.

${ }^{4}$ Manufacturer's score interpretation: reliable identification to the species level.

rectly identified, each with a higher score of 2.382 , and although the second match in both cases was $S$. sciuri, the difference in score between first and second match was greater than with the existing database, as the second match scores did not see the same increase (1.748 and 1.755). Based on the manufacturer's criteria, reliable identification to the species level was achieved by $74.3 \%(168 / 226)$ of the isolates (i.e., $\geq 2.000$; Table 4$)$.

\section{Resolution of Discordant Results}

For the 19 isolates with discordant identification re-submitted for sequencing, perfect agreement was found between rpoB and $h s p 60$, except for one isolate not submitted for rpo $B$ and one isolate that did not meet the sequence homology criteria for $h s p 60$. Three isolates that achieved $\geq 94.0 \%$ sequence homology for $r p o B$ had $<5 \%$ difference from the next bacterial species identified; for these 3 isolates, final reference identification was based on $h s p 60$ results. For all but one isolate, the re-sequencing based identification was in agreement with the MALDI-ToF MS classification. For this collection of 19 isolates, the identifications based upon re-sequencing were used as the reference identification for the evaluation of MALDI-ToF MS. The sequencing results and MALDI-ToF MS identifications are presented in Supplemental Table S2 (https:// doi.org/10.3168/jds.2016-12020).

\section{DISCUSSION}

Matrix-assisted laser desorption/ionization time-offlight mass spectrometry is an accurate, cost-effective, and time-saving method for the routine identification of bacteria (Seng et al., 2009). In this study, we tested a collection of CNS isolates with MALDI-ToF MS and compared the results to a reference gene sequencingbased identification.

In order for an isolate to be identified by MALDIToF MS, it must be represented within the spectral database that is coupled to the instrument. Indeed, comprehensive species coverage is important for all commercial identification systems. An important benefit of MALDI-ToF MS is that users are able to create new entries within the spectral database, thus filling in gaps that may exist within the commercial library. The relative ease of creating new MSP, which can be done by individual laboratories as necessary, enables users of MALDI-ToF MS to maintain an updated database that meets their specific needs even in the changing world of bacteriology. This ability to adapt is not shared by commercially available phenotypic systems that are commonly used in diagnostic bacteriology. The Biotyper database used in the current study did not have entries for $S$. devriesei and S. gallinarum, and as a result, was unable to identify these isolates when relying on the existing commercial database. Addition of 
MSP solved the problem of no identification for these 2 species.

Bruker's identification score interpretation criteria are based on results obtained after following formic acid protein extraction procedures on samples before spotting onto the target. When creating reference spectra, formic acid extraction is used to break down the bacterial cells so that the proteins are exposed and a more fine-tuned spectrum is obtained. Formic acid extraction is a multi-step process and is not considered feasible or practical when using MALDI-ToF MS for routine diagnostic bacteriology (McElvania TeKippe et al., 2013). Whereas the manufacturer's cutoff for specieslevel identification is 2.000 , reducing the cutoff when using a direct transfer protocol improves identification rates with minimal adverse effect on accuracy for grampositive cocci (Schulthess et al., 2013) and more generally for gram-positive bacteria (Mestas et al., 2016). In the current study, lowering the cutpoint to 1.700 would improve species-level identification rates (in this case, from 64 to $92 \%$ for the existing commercial database), with only minimal effect on the accuracy of identification of bovine-associated CNS. For the one isolate of $S$. fleurettii that was misidentified as $S$. sciuri with a score of 1.907, the manufacturer's reporting guidelines would dictate that the result be reported to the genus level only. A reduced species-level cutpoint at 1.700 would result in this sample as being incorrectly identified and potentially reported as $S$. sciuri. However, it is important to note that the result had poor discrimination as the second match was $S$. fleurettii, with a close score of 1.892, and this would be flagged and reported. As a substitute for the full extraction technique, formic acid overlay of a direct smear has been reported to increase identification scores of gram-positive bacteria (Schulthess et al., 2013), but others have concluded that the extra effort does not result in substantially better scoring (McElvania TeKippe et al., 2013; Mestas et al., 2016). Accepting a lower score with a direct smear approach is a reasonable balance between efficiency (i.e., streamlined workflow) and accuracy (Seng et al., 2009).

In addition to simply being present within the database, replication within the database can also be important. Scores $<1.700$ can also occur in species that are contained within the database, but strain differences can be extensive enough to require additional MSP to cover the variability that can exist within a species (Seng et al., 2009). Furthermore, the Biotyper database is predominantly populated by human bacterial isolates, with gradual expansion with veterinary isolates (Tomazi et al., 2014). Previous studies have established a positive association between identification and database redundancy, concluding that multiple reference spectra representing differences in protein expression are required to capture the diversity that exists among the isolates of many species (Seng et al., 2009; Lartigue et al., 2009; Alatoom et al., 2011). In the commercial database used in the current study, $S$. chromogenes was represented by a single MSP. Previous work using high-resolution melt analysis showed $3 \mathrm{~S}$. chromogenes genotypes within the MPCC (Ajitkumar et al., 2013). Similarly, an examination of the genetic diversity among CNS species causing bovine IMI using molecular typing methods revealed 5 genotypes among S. chromogenes identified on 6 dairy farms (Piessens et al., 2012). It was plausible that these genotypes would have different spectra and would require their own entry within the database. By the addition of MSP for 8 species based on both prior knowledge of the species and results of the assessment of the database, we were able to eliminate the unidentified risk (i.e., from $8 \%$ unidentified to $0 \%$ unidentified). However, for S. chromogenes, only a very small overall increase in identification score was achieved in response to the addition of 3 MPSs to the database. Piessens et al. (2012) reported that species heterogeneity for $S$. chromogenes was in fact limited as compared with $S$. haemolyticus, which was represented by 27 different genotypes in the same study of 6 dairy farms. Redundancy in the database is potentially more important for CNS species with greater heterogeneity, such as S. haemolyticus, than for $S$. chromogenes, which is comparatively less diverse. Staphylococcus haemolyticus is represented by 12 MSP in the existing commercial database.

In the current study, for the main classification, all isolates were initially classified in duplicate with a second round of duplicate classification as required for samples that failed to achieve a score $\geq 1.700$ (no reliable identification). Although a single test would reduce analysis time and cost, duplicate tests have been shown to significantly increase the rates of species identification when compared with a single test per sample (Tan et al., 2012; Han et al., 2015). Compared with traditional biochemical methods, MALDI-ToF MS analysis in duplicate still offers a significant cost and time savings (Seng et al., 2009; Tan et al., 2012).

With regard to validation studies of diagnostic technologies, deficiencies in typeability are concerning, but not as concerning as obtaining an incorrect diagnosis. Regarding the performance of MALDI-ToF MS in the current study and the existing commercial database, discordant results were obtained for 2 isolates. In the case of the $S$. fleurettii identified as $S$. sciuri by MALDI-ToF MS, the identification score was 1.907 and the second best match was indeed S. fleurettii with a score of 1.892. Similarly, for the isolate of S. hyicus classified in the preliminary run, the best match was $S$. chromogenes with a score of 2.025 , but the next most 
similar species was S. hyicus with a score of 1.803 . Another classification with poor discrimination occurred for a correctly identified $S$. fleurettii that had a similar scoring second match of $S$. sciuri. Discordant and poor discriminatory results can occur when related bacterial species have very similar spectral profiles, and therefore it is difficult for MALDI-ToF MS to resolve the correct identification. Staphylococcus sciuri and S. fleurettii are both members of the Staphylococcus sciuri species group and are closely related (Nemeghaire et al., 2014). Similarly, S. hyicus and S. chromogenes were once subspecies of the species S. hyicus (Devriese et al., 1978). Improvements to discordant and poor discriminatory results might be made possible by the addition of MSP to the database to capture the differences in protein expression of different strains within the species, as was the case with $S$. fleurettii. With the addition of a single MSP for S. fleurettii, we were able to correct both the misidentification of the one isolate and eliminate the poor discrimination of both isolates. However, in certain cases, the similarity between species is such that distinguishing them is not possible. An example of this is with Streptococcus pneumoniae and other members of the Streptococcus mitis group (Werno et al., 2012; Schulthess et al., 2013). In these cases, the results from the commercial database are flagged with a comment on the difficulty of distinguishing species due to similar patterns.

Regarding the 18 isolates that initially had discordant results between reference and MALDI-ToF MS, but were later resolved in favor of MALDI-ToF MS with further genotypic examination, there are many potential reasons for the inconsistencies between sample label and content (e.g., errors is labeling or mixed growth in stock isolates), and are often a fact when working with a large collection of stored field isolates. In test validation studies, it is important to resolve these ambiguous results using appropriate and adequate care, as was done in the current study. The review and resolution of these discordants provides management strategies for future work, as well as sequence data that will enable further acceptance of MALDI-ToF MS classifications.

Commercial phenotypic identification systems have been traditionally used for CNS species-level diagnosis. Investigations into the accuracy of these systems to identify bovine-associated CNS have demonstrated that they are limited in both species coverage and accuracy (Zadoks and Watts, 2009; Vanderhaeghen et al., 2015). Reports vary with respect to reference identification methods and score criteria, but overall accuracy ranges from $41.3 \%$ for the API Staph ID 32 (API Test, bioMérieux, France) with typeability $\geq 90 \%$ as compared with rpoB (Sampimon et al., 2009) to accuracy of $76.0 \%$ for API Staph ID 20 (API Test, bioMérieux,
France) with typeability $\geq 80 \%$ as compared with $16 \mathrm{~S}$ rRNA (Park et al., 2011). Due to issues with accuracy, according to those who have conducted evaluations of the commercial phenotypic tests, these systems should not be used for the identification of bovine-associated CNS (Capurro et al., 2009; Sampimon et al., 2009; Park et al., 2011).

In contrast, genotypic methods have been shown to perform much better in terms of typeability and accuracy than phenotypic systems (Zadoks and Watts, 2009; Vanderhaeghen et al., 2015). For example, genomic DNA fingerprinting methods have high typeability and accuracy, and share with MALDI-ToF MS the ability to update reference databases with data on additional species and strains as required. For the identification of CNS with user-updated reference databases, transfer RNA-intergenic spacer PCR (tDNA-PCR), amplified fragment length polymorphism (AFLP), and (5'-GTGGTGGTGGTGGTG-3') primer PCR have been shown to be reliable tools, with typeabilities ranging from 91.0\% (tDNA-PCR) to $98.4 \%$ (AFLP) and accuracies ranging from $94.3 \%$ [(5'-GTGGTGGTGGTGGTG-3') primer PCR] to $99.2 \%$ (tDNA-PCR and AFLP; Supré et al., 2009; Piessens et al., 2010; Braem et al., 2011). Based on the results of the current study, MALDI-ToF MS with the custom MSP expanded database achieved similar to moderately better results to the previously validated genotypic methods.

In recent years, the focus on CNS as a mastitis-associated organism has centered on the importance of identifying isolates of CNS to the species level (Zadoks and Watts, 2009; Vanderhaeghen et al., 2015). Considering that previous research has in fact shown that certain CNS species play a larger role in bovine mastitis, causing significant increases in SCC and negatively affecting milk quality, whereas others appear to be simple cohabitants of the teat skin, the development of effective control programs will depend on our ability to make diagnoses to the species level (Vanderhaeghen et al., 2015). In conclusion, MALDI-ToF MS using the direct transfer method was shown to be a highly reliable tool for the identification of bovine-associated CNS. Both the addition of CNS MSP to the existing commercial database and the reduction of the species level cutpoint to $\geq 1.700$ improved the performance of MALDI-ToF MS when classifying CNS isolates. Further validation of the system and the custom MSP expanded database with a larger collection of field isolates is warranted and is the subject of a subsequent study.

\section{ACKNOWLEDGMENTS}

We thank Larissa Condas from the University of Calgary and Anneleen De Visscher from the M-team 
at Ghent University for their contribution to the reference identifications of the CNS. The authors also thank Béatrice Després, Atlantic Veterinary College MALDI-TOF mass spectrometry Classification Research \& Development Unit, and Natasha Robinson, Maritime Quality Milk (University of Prince Edward Island, Charlottetown, Canada), for their technical assistance. This research was supported by the Dairy Research Cluster initiative (Dairy Farmers of Canada, Agriculture and Agri-Food Canada, and the Canadian Dairy Commission) through the Canadian Bovine Mastitis and Milk Quality Research Network (Université de Montréal, Saint-Hyacinthe, Canada).

\section{REFERENCES}

Ajitkumar, P., H. W. Barkema, R. N. Zadoks, D. W. Morck, F. J. U. M. van der Meer, and J. De Buck. 2013. High-resolution melt analysis for species identification of coagulase-negative staphylococci derived from bovine milk. Diagn. Microbiol. Infect. Dis 75:227-234. https://doi.org/10.1016/j.diagmicrobio.2012.11.008.

Alatoom, A. A., S. A. Cunningham, S. M. Ihde, J. Mandrekar, and R. Patel. 2011. Comparison of direct colony method versus extraction method for identification of Gram-positive cocci by use of Bruker Biotyper Matrix-Assisted Laser Desorption Ionization-Time of Flight mass spectrometry. J. Clin. Microbiol. 49:2868-2873. https://doi.org/10.1128/JCM.00506-11.

Bellamy, J. E. C., and D. W. Olexson. 2000. Quality Assurance Handbook for Veterinary Laboratories. Iowa State University Press, Ames.

Braem, G., S. De Vliegher, K. Supré, F. Haesebrouck, F. Leroy, and L. De Vuyst. 2011. (GTG)5-PCR fingerprinting for the classification and identification of coagulase-negative Staphylococcus species from bovine milk and teat apices: a comparison of type strains and field isolates. Vet. Microbiol. 147:67-74. https://doi.org/10.1016/j. vetmic.2010.05.044.

Capurro, A., K. Artursson, K. Waller, B. Bengtsson, H. Ericssonunnerstad, and A. Aspan. 2009. Comparison of a commercialized phenotyping system, antimicrobial susceptibility testing, and tuf gene sequence-based genotyping for species-level identification of coagulase-negative staphylococci isolated from cases of bovine mastitis. Vet. Microbiol. 134:327-333. https://doi.org/10.1016/j. vetmic.2008.08.028.

Devriese, L. A., V. Hajek, P. Oeding, S. A. Meyer, and K. H. Schleifer. 1978. Staphylococcus hyicus (Sompolinsky 1953) comb. nov. and Staphylococcus hyicus ssp. chromogenes ssp. nov. Int. J. Syst. Evol. Microbiol. 28:482-490.

Halasa, T., K. Huijps, O. Østerås, and H. Hogeveen. 2007. Economic effects of bovine mastitis and mastitis management: A review. Vet. Q. 29:18-31. https://doi.org/10.1080/01652176.2007.9695224.

Han, H. W., H. C. Chang, A. H. Hunag, and T. C. Chang. 2015. Optimization of the score cutoff value for routine identification of Staphylococcus species by Matrix-Assisted Laser Desorption Ionization-Time-of-Flight mass spectrometry. Diagn. Microbiol. Infect. Dis. 83:349-354. https://doi.org/10.1016/j. diagmicrobio.2015.08.003.

Kliem, M., and S. Sauer. 2012. The essence on mass spectrometry based microbial diagnostics. Curr. Opin. Microbiol. 15:397-402. https://doi.org/10.1016/j.mib.2012.02.006.

Lartigue, M.-F., G. Hery-Arnaud, E. Haguenoer, A.-S. Domelier, P.O. Schmit, N. van der Mee-Marquet, P. Lanotte, L. Mereghetti, M. Kostrzewa, and R. Quentin. 2009. Identification of Streptococcus agalactiae isolates from various phylogenetic lineages by Matrix-Assisted Laser Desorption Ionization-Time of Flight mass spectrometry. J. Clin. Microbiol. 47:2284-2287. https://doi. org/10.1128/JCM.00175-09.
Makovec, J. A., and P. L. Ruegg. 2003. Antimicrobial resistance of bacteria isolated from dairy cow milk samples submitted for bacterial culture: 8,905 samples (1994-2001). J. Am. Vet. Med. Assoc. $222: 1582-1589$.

McElvania TeKippe, E., S. Shuey, D. W. Winkler, M. A. Butler, and C.-A. D. Burnham. 2013. Optimizing identification of clinically relevant Gram-positive organisms by use of the Bruker Biotyper Matrix-Assisted Laser Desorption Ionization-Time of Flight mass spectrometry system. J. Clin. Microbiol. 51:1421-1427. https:// doi.org/10.1128/JCM.02680-12.

Mellmann, A., K. Becker, C. Von Eiff, U. Keckevoet, P. Schumann, and D. Harmsen. 2006. Sequencing and staphylococci identification. Emerg. Infect. Dis. 12:333-336.

Mestas, J., T. Quias, and J. Dien Bard. 2016. Direct identification of aerobic bacteria by Matrix-Assisted Laser Desorption Ionization Time-of-Flight mass spectrometry is accurate and robust. J. Clin. Lab. Anal. 30:543-551. https://doi.org/10.1002/jcla.21900.

Nemeghaire, S., M. A. Argudín, A. T. Feßler, T. Hauschild, S. Schwarz, and P. Butaye. 2014. The ecological importance of the Staphylococcus sciuri species group as a reservoir for resistance and virulence genes. Vet. Microbiol. 171:342-356. https://doi. org/10.1016/j.vetmic.2014.02.005.

Park, J. Y., L. K. Fox, K. S. Seo, M. A. McGuire, Y. H. Park, F. R. Rurangirwa, W. M. Sischo, and G. A. Bohach. 2011. Comparison of phenotypic and genotypic methods for the species identification of coagulase-negative staphylococcal isolates from bovine intramammary infections. Vet. Microbiol. 147:142-148. https://doi. org/10.1016/j.vetmic.2010.06.020.

Piepers, S., L. De Meulemeester, A. de Kruif, G. Opsomer, H. W. Barkema, and S. De Vliegher. 2007. Prevalence and distribution of mastitis pathogens in subclinically infected dairy cows in Flanders, Belgium. J. Dairy Res. 74:478-483. https://doi.org/10.1017/ S0022029907002841.

Piessens, V., S. De Vliegher, B. Verbist, G. Braem, A. Van Nuffel, L. De Vuyst, M. Heyndrickx, and E. Van Coillie. 2012. Intra-species diversity and epidemiology varies among coagulase-negative staphylococcus species causing bovine intramammary infections. Vet. Microbiol. 155:62-71. https://doi.org/10.1016/j.vetmic.2011.08.005.

Piessens, V., K. Supré, M. Heyndrickx, F. Haesebrouck, S. De Vliegher, and E. Van Coillie. 2010. Validation of amplified fragment length polymorphism genotyping for species identification of bovine associated coagulase-negative staphylococci. J. Microbiol. Methods 80:287-294. https://doi.org/10.1016/j.mimet.2010.01.012.

Pitkälä, A., M. Haveri, S. Pyörälä, V. Myllys, and T. Honkanen-Buzalski. 2004. Bovine mastitis in Finland 2001-Prevalence, distribution of bacteria, and antimicrobial resistance. J. Dairy Sci 87:2433-2441.

Reyher, K. K., S. Dufour, H. W. Barkema, L. Des Côteaux, T. J. DeVries, I. R. Dohoo, G. P. Keefe, J.-P. Roy, and D. T. Scholl 2011. The National Cohort of Dairy Farms - A data collection platform for mastitis research in Canada. J. Dairy Sci. 94:16161626. https://doi.org/10.3168/jds.2010-3180.

Royster, E., and S. Wagner. 2015. Treatment of mastitis in cattle. Vet. Clin. North Am. Food Anim. Pract. 31:17-46. https://doi. org/10.1016/j.cvfa.2014.11.010.

Ruegg, P. L. 2012. New Perspectives in udder health management Vet. Clin. North Am. Food Anim. Pract. 28:149-163. https://doi. org/10.1016/j.cvfa.2012.03.001.

Sampimon, O. C., T. J. G. M. Lam, D. J. Mevius, Y. H. Schukken, and R. N. Zadoks. 2011. Antimicrobial susceptibility of coagulase-negative staphylococci isolated from bovine milk samples. Vet. Microbiol. 150:173-179. https://doi.org/10.1016/j.vetmic.2011.01.017.

Sampimon, O. C., R. N. Zadoks, S. De Vliegher, K. Supré, F. Haesebrouck, H. W. Barkema, J. Sol, and T. J. G. M. Lam. 2009. Performance of API Staph ID 32 and Staph-Zym for identification of coagulase-negative staphylococci isolated from bovine milk samples. Vet. Microbiol. 136:300-305. https://doi.org/10.1016/j. vetmic.2008.11.004.

Schulthess, B., K. Brodner, G. V. Bloemberg, R. Zbinden, E. C. Bottger, and M. Hombach. 2013. Identification of Gram-positive cocci by use of Matrix-Assisted Laser Desorption Ionization-Time of 
Flight mass spectrometry: Comparison of different preparation methods and implementation of a practical algorithm for routine diagnostics. J. Clin. Microbiol. 51:1834-1840. https://doi. org/10.1128/JCM.02654-12.

Seng, P., M. Drancourt, F. Gouriet, B. La Scola, P. Fournier, J. M. Rolain, and D. Raoult. 2009. Ongoing revolution in bacteriology: Routine identification of bacteria by Matrix-Assisted Laser Desorption Ionization Time-of-Flight mass spectrometry. Clin. Infect. Dis. 49:543-551. https://doi.org/10.1086/600885.

Supré, K., S. De Vliegher, O. C. Sampimon, R. N. Zadoks, M. Vaneechoutte, M. Baele, E. De Graef, S. Piepers, and F. Haesebrouck. 2009. Technical note: Use of transfer RNA-intergenic spacer PCR combined with capillary electrophoresis to identify coagulase-negative Staphylococcus species originating from bovine milk and teat apices. J. Dairy Sci. 92:3204-3210. https://doi. org/10.3168/jds.2008-1923.

Supré, K., F. Haesebrouck, R. N. Zadoks, M. Vaneechoutte, S. Piepers, and S. De Vliegher. 2011. Some coagulase-negative Staphylococcus species affect udder health more than others. J. Dairy Sci. 94:2329-2340. https://doi.org/10.3168/jds.2010-3741.

Tan, K. E., B. C. Ellis, R. Lee, P. D. Stamper, S. X. Zhang, and K. C. Carroll. 2012. Prospective evaluation of a MALDI-TOF MS system in a hospital clinical microbiology laboratory for the identification of bacteria and yeasts: A bench-by-bench study to assess the impact on time-to-identification and cost-effectiveness. J. Clin. Microbiol. 50:3301-3308. http://dx.doi.org/https://doi. org/10.1128/JCM.01405-12.
Tomazi, T., J. L. Gonçalves, J. R. Barreiro, P. A. de Campos Braga, L. F. Prada e Silva, M. N. Eberlin, and M. V. dos Santos. 2014. Identification of coagulase-negative staphylococci from bovine intramammary infection by Matrix-Assisted Laser Desorption Ionization-Time of Flight mass spectrometry. J. Clin. Microbiol. 52:1658-1663. https://doi.org/10.1128/JCM.03032-13.

Vanderhaeghen, W., S. Piepers, F. Leroy, E. Van Coillie, F. Haesebrouck, and S. De Vliegher. 2014. Invited review: Effect, persistence, and virulence of coagulase-negative Staphylococcus species associated with ruminant udder health. J. Dairy Sci. 97:5275-5293. https://doi.org/10.3168/jds.2013-7775.

Vanderhaeghen, W., S. Piepers, F. Leroy, E. Van Coillie, F. Haesebrouck, and S. De Vliegher. 2015. Identification, typing, ecology and epidemiology of coagulase negative staphylococci associated with ruminants. Vet. J. 203:44-51. https://doi.org/10.1016/j. tvjl.2014.11.001.

Werno, A. M., M. Christner, T. P. Anderson, and D. R. Murdoch. 2012. Differentiation of Streptococcus pneumoniae from nonpneumococcal streptococci of the Streptococcus mitis group by Matrix-Assisted Laser Desorption Ionization-Time of Flight mass spectrometry. J. Clin. Microbiol. 50:2863-2867. https://doi. org/10.1128/JCM.00508-12.

Zadoks, R. N., and J. L. Watts. 2009. Species identification of coagulase-negative staphylococci: Genotyping is superior to phenotyping. Vet. Microbiol. 134:20-28. https://doi.org/10.1016/j. vetmic.2008.09.012 